\title{
Low-voltage electrostatic modulation of ion diffusion through layered graphene-based nanoporous membranes
}

Chi Cheng ${ }^{1,2}$, Gengping Jiang ${ }^{3,4}$, George Philip Simon ${ }^{2}$, Jefferson Zhe Liu ${ }^{5,6^{*}}$ and Dan $\mathrm{Li}^{1,2^{*}}$

${ }^{1}$ Department of Chemical Engineering, University of Melbourne, VIC 3010, Australia;

${ }^{2}$ Department of Materials Science and Engineering and New Horizons Research Centre, Monash University, VIC 3800, Australia; ${ }^{3}$ College of Science, Wuhan University of Science and Technology, Wuhan 430081, China; ${ }^{4}$ The State Key Laboratory of Refractories and Metallurgy, Hubei Province Key Laboratory of Systems Science on Metallurgical Processing, Wuhan University of Science and Technology, Wuhan 430081, China ${ }^{5}$ Department of Mechanical Engineering, University of Melbourne, VIC 3010, Australia; ${ }^{6}$ Department of Mechanical and Aerospace Engineering, Monash University, VIC 3800, Australia

Corresponding author Email: dan.li1@unimelb.edu.au (D.L.); zhe.liu@unimelb.edu.au Ion transport in nanoconfinement differs from that in bulk, has been extensively researched across scientific and engineering disciplines ${ }^{1,2,3,4}$. For many energy and water applications of nanoporous materials, concentration-driven ion diffusion is simultaneously subjected to a local electric field arising from surface charge or an externally applied potential. Due to the uniquely crowded intermolecular forces under severe nanoconfinement $(<2 \mathrm{~nm})$, the transport behaviours of ions can be influenced by the interfacial electrical double layer (EDL) induced by a surface potential, with complex implications, engendering unusual ion dynamics ${ }^{5,6,7}$. However, it remains an experimental challenge to investigate how such a surface potential and its coupling with nanoconfinement manipulate ion diffusion. Here, we exploit the tuneable 
nanoconfinement in layered graphene-based nanoporous membranes, to show that subdiffusion rates can be reversibly modulated and anomalously enhanced by $4 \sim 7$ times within 0.5 volts, across a salt concentration gradient up to seawater salinity. Modelling suggests that this anomalously enhanced diffusion be related to the strong ion-ion correlations under severe nanoconfinement, and cannot be explained by conventional theoretical predictions.

The recent development in two-dimensional (2D) nanofluidics ${ }^{8,9}$, featuring graphene, 42 graphene-based and other atomically thin materials, has enabled severe nanoconfinement tuneable down to subnanometer level to be produced, giving rise to a plethora of anomalous 44 transport phenomena such as ultrafast and precise molecular selectivity ${ }^{10,11,12}$. We have previously shown that by the supramolecular assembly of chemically converted graphene, a layered, nanoporous gel membrane (Fig. 1a) can be readily produced, containing a

47 continuous cascading nanochannel network ${ }^{13}$. The average interlayer spacing, $d$, of the membranes can be tuned in the range of a few to sub-nanometres ${ }^{14}$. Under such a severe nanoconfinement $(<2 \mathrm{~nm})$, it should contain almost entirely an interfacial electrical double

50 layer (EDL) without bulk solution between any two graphene layers, even if the salt concentration is up to $0.1 \mathrm{M}$. Furthermore, electrically conducting graphene materials can act

52 simultaneously as a channel wall and a gate electrode. This allows for effective modulation of

53 the interfacial EDL electrostatically in-situ, without the need for an additional dielectric layer

54 of a channel wall made with, for example, silicon-based materials as used in conventional

55 devices such as field-effect nanofluidic transistors ${ }^{15}$. Due to the exceptionally large EDL

56 capacitance of chemically converted graphene ${ }^{16}$, a significant change to the interfacial double 
57 layer ion population and distribution can be readily achieved by applying a very low surface

58 potential $(<1 \mathrm{~V})$. Additionally, since the membrane is a multi-channel platform, a much

59 higher flux is possible compared to single channel devices, allowing ready detection of

60 permeate signals within a time scale of a few minutes ${ }^{14}$. This means that layered graphene-

61 based nanoporous membranes represent a unique experimental platform upon which ion

62 diffusion through a barrier of nanoconfined EDL can be studied.

63

64 We used the experiment setup shown in Fig. 1a to examine how concentration-driven ion

65 diffusion is influenced by the EDL induced by varying the surface potential in a confined

66 nanochannel using the layered graphene-based nanoporous membrane. Since the membrane

67 is both mechanically self-supporting and electrically conductive, a gate potential $\left(\mathrm{V}_{\mathrm{g}}\right)$ was

68 applied directly to the membrane to tune the EDL enclosed between the layers of graphene

69 materials. A concentration gradient was then created to drive ions to diffuse through the

70 series of cascading graphene nanochannels in the membranes with the interlayer spacing

71 varied from $5.4 \mathrm{~nm}$ to $0.8 \mathrm{~nm}$ (Supplementary Fig. 1). Such a design allows for the study of

72 ion diffusion, recorded as diffusion flux $(J)$, through nanoconfined EDL, whose structure can

73 be tuned in-situ by an externally applied potential or the degree of nanoconfinement (i.e. the

74 interlayer spacing).

75

76 Figure $1 \mathrm{~b}$ shows the diffusion of $\mathrm{KCl}$ through the layered graphene membrane under a

77 constant $V_{g}$, whereby the diffusion rates were significantly changed with different $V_{g}$.

78 Specifically, as the $\mathrm{V}_{\mathrm{g}}$ was changed from $-0.2 \mathrm{~V}$ to $-0.5 \mathrm{~V}$, diffusion fluxes were enhanced by

79 approximately four times from $7.93 \times 10^{-3}$ to $3.02 \times 10^{-2} \mathrm{~mol} \mathrm{~h}^{-1} \mathrm{~m}^{-2}$. By contrast, only a slight

80 flux enhancement was observed when the membrane was positively charged up to $0.5 \mathrm{~V}$

81 (Supplementary Fig. 2a). This asymmetry between $\mathrm{K}^{+}$and $\mathrm{Cl}^{-}$is not uncommon under 
82 nanoconfinement, although they exhibit the same diffusion coefficient and mobilities in bulk

83 solutions. Due to the different water polarisation around cations and anions, $\mathrm{K}^{+}$gives higher

84 EDL capacitance than $\mathrm{Cl}^{-}$on graphene surface ${ }^{17}$. As such, the channel ion populations

85 experience an asymmetric rearrangement as the charging status of the membrane shifts from

86 positive to negative polarisation. Flux responses to immediate changes in the $\mathrm{V}_{\mathrm{g}}$ were further

87 investigated and the results are shown in Fig. 1c. Ion permeation rates across the layered

88 graphene membrane were spontaneously changed, depending on the $V_{g}$ applied, as seen by

89 the sharp gradient change of the diffusion curves. Cycling of the $\mathrm{V}_{\mathrm{g}}$ between $-0.5 \mathrm{~V}$ and 0.2

$90 \mathrm{~V}$, and subsequent comparison of fluxes measured at each voltage showed that such an

91 electrostatic modulation effect of ion transport through the membrane was reversible (Fig.

92 1d). The flux change during one $V_{g}$ shift was completed within a timescale of tens of

93 seconds. We further confirmed that the enhancement of $J$ with increasing $\mathrm{V}_{\mathrm{g}}$ was mainly

94 resulted from the change of the graphene-enclosed EDL structure by ruling out the influence

95 from membrane expansion (Supplementary Figs. 2b and c) and water oxidation/reduction

96 (Supplementary Fig. 3) during the diffusion tests. Ion concentrations in the permeate

97 compartment at the end of diffusion tests were also independently analysed using inductively

98 coupled plasma mass spectrometry. The results are in good agreement with those derived

99 from the solution conductivity analysis (Supplementary Fig. 4).

101 The critical role of severe nanoconfinement is shown by displaying the full $J-\mathrm{V}_{\mathrm{g}}$ 102 relationships in which the ion fluxes at different $\mathrm{V}_{\mathrm{g}}$ were normalised to that of $\mathrm{V}_{\mathrm{g}}=0 \mathrm{~V}$ (Figs. 103 2a-c). The curve profile was asymmetrically parabolic, with a sharp increase in flux values as 104 the $\mathrm{V}_{\mathrm{g}}$ became more negative to $-0.5 \mathrm{~V}$, and through membranes with $d$ smaller than $2 \mathrm{~nm}$ 105 (black and orange curves in Fig. 2a). The normalized ion flux was three times higher through 106 a membrane with $d=0.8 \mathrm{~nm}$ than a membrane with $d=5.4 \mathrm{~nm}$, as the $\mathrm{V}_{\mathrm{g}}$ was increased from 
1070 to $-0.5 \mathrm{~V}$, and measured for the same feed concentration of $0.1 \mathrm{M}$. In the cases of greater $d$,

108 the modulation of ion diffusion was reduced. Through a membrane with the largest

109 experimented $d$ of $5.4 \mathrm{~nm}$, the enhancement of ion flux diminished, and the flux even

110 exhibited a slight decrease as $\mathrm{V}_{\mathrm{g}}$ approached either $\pm 0.5 \mathrm{~V}$. This is consistent with a recent

111 report that the rate of ion diffusion through a mesoporous carbon membranes with a pore size

112 of $\sim 7.8 \mathrm{~nm}$ remained unchanged when a gate potential of up to $-0.8 \mathrm{~V}$ was applied $^{18}$.

114 Further investigation on the dependence of modulation ratio on concentration gradient (Fig.

115 2) suggested that the mechanism of such modulated diffusion is closely related to the 116 structural variation of nanoconfined EDL. As the ion concentration in the feed reservoir was 117 increased, and the EDL would be thinner and more compact to the surface, the modulation of 118 ion diffusion was reduced and diminished when the feed concentration reached $0.5 \mathrm{M}$, even 119 when the channel size is as small as $0.8 \mathrm{~nm}$. In cases where the membrane was positively 120 charged, a slight flux increase was also observed. This asymmetric $J-\mathrm{V}_{\mathrm{g}}$ relationship 121 depending on the sign of surface potential applied indicates the essential difference between 122 the EDL structures in which either $\mathrm{K}^{+}$or $\mathrm{Cl}^{-}$were the counter ions.

123

124 Such enhanced ion diffusion through the EDL induced with increased surface potential under 125 nanoconfinement of less than $2 \mathrm{~nm}$ is contrary to the predictions based on the classical ion 126 transport theory ${ }^{2}$. Our simulation study based on the classic Poisson-Nernst-Planck (PNP) 127 model $^{19}$, showed that diffusion flux indeed should decrease through the EDL confined in 128 nanochannels with a size of $2 \mathrm{~nm}$ with the increase of gate potential (Supplementary Figs. 5 129 and 6). The results revealed significant co-ion depletion in the overlapping EDL structure 130 confined between the graphene layers, with an increased surface potential (inset in 131 Supplementary Fig. 6). The decreased co-ion concentration in EDL lead to an accumulation 
132 of counter-ions in the drain side and thus a build-up of the Donnan potential. Such an

133 potential, once established, will reduce the counter-ion flux to match the co-ion flux. Further

134 investigations on other possible factors, including the medium dielectric constant

135 (Supplementary Fig. 6), and an increased ion diffusion coefficient (Supplementary Fig. 7),

136 which are known to vary under severe nanoconfinement, also confirmed a prohibited

137 diffusion under increased surface potential, consistent with the Teorell-Meyer-Sievers or

138 space-charge models for nanofiltration membrane ${ }^{20}$. Such a reduced ion flux through the

139 unipolar EDL has indeed previously been used for salt rejection ${ }^{21}$, and to control membrane 140 selectivity ${ }^{22}$

142 Similar anomalous, electrostatically-modulated ion diffusion phenomena under extreme 143 nanoconfinement were also observed with both monovalent and bivalent cation/anion pairs, 144 though the degree of modulated diffusion was ion specific. Among the monovalent and 145 bivalent cation/anion pairs studied, the degree of modulated diffusion through a membrane 146 with $d$ of $2 \mathrm{~nm}$ was found to reach a factor of over 6 for $\mathrm{K}_{2} \mathrm{SO}_{4}$, nearly double the modulation 147 ratio of $\mathrm{KCl}$ within the same $\mathrm{V}_{\mathrm{g}}$ ranging from 0 to $-0.5 \mathrm{~V}$, while negligible modulation was 148 seen for $\mathrm{MgCl}_{2}$ (Fig. 3a). When $\mathrm{Cl}^{-}$was selected as the anion and paired with $\mathrm{Li}^{+}, \mathrm{Na}^{+}, \mathrm{K}^{+}$ 149 and $\mathrm{Cs}^{+}$, respectively, ion diffusion through the layered graphene membranes was enhanced, 150 yet exhibited insignificant ion specificity, and a modulation ratio approaching four under a $\mathrm{V}_{\mathrm{g}}$ 151 of $-0.5 \mathrm{~V}$, compared with a $\mathrm{V}_{\mathrm{g}}$ of $-0.2 \mathrm{~V}$ (Supplementary Fig. 8). In addition, if alkali cations 152 were paired with molecular anions of weak carboxylic acids, which are commonly present on 153 the surfaces of intra- and extracellular proteins, the modulated diffusion could be observed, 154 even under a feed concentration up to $0.5 \mathrm{M}$ (Fig. 3b). Importantly, the contrast between 
155 sodium acetate and sodium chloride indicates the significant role the specific noncovalent

156 interactions between hydrated cations and anions plays in the modulation mechanism.

157

158 To develop a more realistic model to elucidate this unexpected phenomena, we considered the

159 role of the short-range, ion-specific ion-ion correlations in enhancing the ion diffusion under

160 extreme nanoconfinement and a surface potential. Recent developments suggest that the non-

161 Coulombic-form, ion-ion correlations ${ }^{23}$ can contribute considerably to the alternation of ion

162 arrangements of the EDL, particularly in the first few ion layers closely confined to the

163 electrode/electrolyte interface. Instead of an ideal, exponential decay of ion concentrations in

164 the EDL from the surface towards the nanochannel centre, an oscillating counter- and co-ion

165 concentration profiles emerge where there can be a peak of much concentrated co-ion layer as

166 a result of such ion-ion correlations. This interaction among counter- and co-ions has become

167 crucial in describing unconventional interfacial phenomenon e.g. "overscreening" 24 and

168 "charge inversion" $"$. Considering the extreme nanoconfinement electrolytes experience in a

169 sub-2 nm graphene nanochannel (<10 layers of water molecules) as in our experiment, it is

170 possible that such ion-ion correlations cannot be completely neglected. We incorporated the

171 ion-ion correlations into the PNP model (later referred to as PNP/IC model), according to

172 Bazant's work ${ }^{24}$ (Supplementary Eq. 4-5), and the geometry of the simulation model

173 illustrated in the inset of Fig. 4d.

174

175 Simulation with the effect of ion-ion correlations (PNP/IC) considered showed that the

176 channel concentrations, especially the co-ion concentrations, markedly increased after

177 demonstrating a minimum with a higher surface potential, which was in contrast to what was

178 revealed with the conventional PNP model (hollow symbols in Fig. 4a). Instead of co-ion

179 depletion traditionally expected for electrostatically charged EDL under nanoconfinement, 

the PNP/IC model predicted an unexpected increase in co-ion concentration with increasing

181 the surface potential. This would lead to the build-up of Donnan potential being hindered, 182 which was confirmed by experiment (Fig. 4b), resulting in an enhanced ion flux as seen in 183 Figure 4c (solid) for the $J-\mathrm{V}_{\mathrm{g}}$ relationship. The trend that weakened diffusion enhancement 184 in channel with larger sizes could also be predicted with the PNP/IC model. Shown in Fig. $1854 \mathrm{~d}$, as the channel size decreases, an increase in the level of enhanced diffusion is observed. 186 Furthermore, the magnitude of increase in the relative flux with $\mathrm{V}_{\mathrm{g}}$ is greater for lower feed 187 concentrations $(50 \mathrm{mM})$ than a higher one $(0.5 \mathrm{M})$, which is also consistent with 188 experimental observations.

190 Although ion-ion correlations have long been acknowledged in colloidal and electrochemical 191 systems $^{23,26}$, their influence on ion transport properties has been insignificant and often 192 neglected in traditional nanoporous materials systems. Our results suggest that such 193 correlations among ions could play a key role in modulating the ion diffusion behaviours if 194 the nanoconfinement, materials and nanoporous structure are appropriately designed. As an 195 example, choosing $\mathrm{SO}_{4}{ }^{2-}$ as co-ions leads to a more significant degree of modulated diffusion 196 as a result of its stronger ion-ion correlations with cations ${ }^{27}$. Under the nanoconfinement of $<$ $1972 \mathrm{~nm}$, a significant drop in the medium dielectric constant (from 78 to 10 for aqueous 198 medium) is expected ${ }^{28}$, affording a much stronger effect of ion-ion correlations on diffusion 199 as a weakening shielding from the dielectric medium (Supplementary Fig. 10). Additionally, 200 the large double layer capacitance of the cascading nanoslits in layered graphene membranes 201 enables a strong manipulation of the overlapping EDL structure, and translates its influence 202 on ion diffusion at the nanoscale to an readily observable macroscopic modulation 203 phenomena using a relative low surface potential. 
205 The ability to effectively modulate the transport of charge carriers in materials by a small 206 external voltage, particularly at the nanoscale, has enabled important technologies such as

207 modern electronics which is largely built on the field effect in semiconducting materials. It

208 has been attempted, as yet with limited success, to extend the use of the electric field to 209 modulate ion transport in nanochannels ${ }^{29}$. Given there are few materials choices currently 210 available for creating extreme nanoconfinement at the molecular level, it has been difficult to 211 determine whether and how the fundamental diffusion of ionic species in liquids can be 212 effectively modulated with a small potential similar to the field effect in solid semiconducting 213 materials. This mechanism of strongly modulated nanoconfined ion diffusion with a low 214 surface potential could enable effective, fast modulation of the diffusion of ionic species 215 within a voltage range compatible with physiological concentrations and environments, such 216 as rapid and selective dialysis and controlled release of ionic drugs. Given the nature of

217 molecular interactions in extreme nanoconfinement coupling with electric fields is complex, 218 the observed modulated ion transport behaviour and the peculiar ion specificity shows 219 promise for researching into the electric field effect in nanoconfined liquid materials, which 220 remains largely unexplored. With the advancement in engineering and scale up production of, 221 atomically-precise nano-pores, -channels and -circuits, field-effect control of ionic transport 222 associated with size reduction is likely to enable logic and signalling machineries and 223 devices, beyond conventional use of nanoionics in energy storage, separation and water 224 desalination.

\section{References}

227 1. Bocquet, L., Charlaix, E. Nanofluidics, from bulk to interfaces. Chem. Soc. Rev. 39, 1073-1095 (2010). 
230

231

232

233

234

235

236

237

238

239

240

241

242

243

244

245

246

247

248

249

250

251

252

253

254

255

256

257

258

259

260

261

262

263

264

265

266

267

268

269

270

271

272

273

274

275

276

277

2. Sparreboom, W., van den Berg, A., Eijkel, J. C .T. Principles and applications of nanofluidic transport. Nat. Nanotechnol. 4, 713-720 (2009).

3. Duan, C., Majumdar, A. Anomalous ion transport in 2-nm hydrophilic nanochannels. Nat. Nanotechnol. 5, 848-852 (2010).

4. Maier, J. Nanoionics: ion transport and electrochemical storage in confined systems. Nat. Mater. 4, 805-815 (2005).

5. Feng, J. et al. Observation of ionic Coulomb blockade in nanopores. Nat. Mater. 15, 850-855 (2016).

6. Griffin, J. M. et al. In situ NMR and electrochemical quartz crystal microbalance techniques reveal the structure of the electrical double layer in supercapacitors. Nat. Mater. 14, 812-819 (2015).

7. Kondrat, S., Wu, P., Qiao, R., Kornyshev, A. A. Accelerating charging dynamics in subnanometre pores. Nat. Mater. 13, 387-393 (2014).

8. Koltonow, A. R., Huang, J. Two-dimensional nanofluidics. Science 351, 1395-1396 (2016).

9. Gao, J., Feng, Y., Guo, W., Jiang, L. Nanofluidics in two-dimensional layered materials: inspirations from nature. Chem. Soc. Rev. 46, 5400-5424 (2017).

10. Joshi, R. K. et al. Precise and Ultrafast Molecular Sieving Through Graphene Oxide Membranes. Science 343, $752-754$ (2014).

11. Morelos-Gomez, A. et al. Effective $\mathrm{NaCl}$ and dye rejection of hybrid graphene oxide/graphene layered membranes. Nat. Nanotechnol. 12, 1083-1088 (2017)

12. Chen, L. et al. Ion sieving in graphene oxide membranes via cationic control of interlayer spacing. Nature 550 , 380-383 (2017).

13. Yang, X. et al. Ordered Gelation of Chemically Converted Graphene for NextGeneration Electroconductive Hydrogel Films. Angew. Chem. In. Ed. 50, 7325-7328 (2011).

14. Cheng, C. et al. Ion transport in complex layered graphene-based membranes with tuneable interlayer spacing. Sci. Adv. 2, e1501272 (2016).

15. Karnik, R. et al. Electrostatic Control of Ions and Molecules in Nanofluidic Transistors. Nano Lett. 5, 943-948 (2005).

16. Yang, X., Cheng, C., Wang, Y., Qiu, L., Li, D. Liquid-Mediated Dense Integration of Graphene Materials for Compact Capacitive Energy Storage. Science 341, 534-537 (2013). 
290

291

292

293

294

295

296

297

298

299

300

301

302

303

304

305

306

307

308

309

310

311

312

313

314

315

316

317

318

319

320

321

322 323

17. Jiang, G., Cheng, C., Li, D., Liu, J. Z. Molecular dynamics simulations of the electric double layer capacitance of graphene electrodes in mono-valent aqueous electrolytes. Nano Res. 9, 174-186 (2016).

18. Surwade, S. P. et al. Electrochemical Control of Ion Transport through a Mesoporous Carbon Membrane. Langmuir 30, 3606-3611 (2014).

19. Borukhov, I., Andelman, D., Orland, H. Steric Effects in Electrolytes: A Modified Poisson-Boltzmann Equation. Phys. Rev. Lett. 79, 435-438 (1997).

20. Schaefer, A., Fane, A. G., Waite, T. Nanofiltration: Principles and Applications 2nd Ed. (2017).

21. Tsuru, T., Nakao, S. I., Kimura, S. Calculation of Ion Rejection by Extended Nernst\&ndash;Planck Equation with Charged Reverse Osmosis Membranes for Single and Mixed Electrolyte Solutions. J. Chem. Eng. JP 24, 511-517 (1991).

22. Nishizawa, M., Menon, V. P., Martin, C. R. Metal Nanotubule Membranes with Electrochemically Switchable Ion-Transport Selectivity. Science 268, 700-702 (1995).

23. Yan, L. Electrostatic correlations: from plasma to biology. Rep. Progr. Phys. 65, 1577 (2002).

24. Bazant, M. Z., Storey, B. D., Kornyshev, A. A. Double layer in ionic liquids: overscreening versus crowding. Phys. Rev. Lett. 106, 046102 (2011).

25. Grosberg, A. Y., Nguyen, T., Shklovskii, B. Colloquium: the physics of charge inversion in chemical and biological systems. Rev. moder. phys. 74, 329 (2002).

26. Quesada-Pérez, M., González-Tovar, E., Martín-Molina, A., Lozada-Cassou, M., Hidalgo-Álvarez, R. Overcharging in colloids: beyond the poisson-boltzmann approach. ChemPhysChem 4, 234-248 (2003).

27. van der Vegt, N. F. A. et al. Water-Mediated Ion Pairing: Occurrence and Relevance. Chem. Rev. 116, 7626-7641 (2016).

28. Ballenegger, V., Hansen, J. P. Dielectric permittivity profiles of confined polar fluids. J. Chem. Phys. 122, 114711 (2005).

29. Prakash, S., Conlisk, A. T. Field effect nanofluidics. Lab Chip 16, 3855-3865 (2016).

\section{Acknowledgements}

We would like to acknowledge the financial support from the Australia Research Council. This work made use of the facilities at the Monash Centre for Electron Microscopy (MCEM). 


\section{Author contribution statement}

325 C.C. conceived, designed, carried out the experiments under the guidance of D.L. and 326 G.P.S. D.L. and C.C. formulated the concept of using nano-confined electrical double layer 327 for ion modulation. G.J. designed and carried out the theoretical modelling under the 328 guidance of J.Z.L. All authors discussed and interpreted the results. C.C. and G.J. wrote the 329 manuscript with contributions from all the other authors.

\section{Competing interests}

332 The authors declare no competing interests

333

334 Figure captions

335 Fig. 1: Ion diffusion through nanoconfined EDLs in charged layered graphene-based 336 nanoporous membranes. a, Schematic showing the experiment step-up for the investigation 337 of ion diffusion $(0.05 \mathrm{M} \mathrm{KCl})$ through nanoconfined EDLs in charged, layered graphene338 based nanoporous membranes with a $d=2 \mathrm{~nm}$. Under such severe nanoconfinement, the 339 electrical double layer, highlighted in bright red and yellow colours, fully fills the membrane. 340 Potentials versus a $\mathrm{Ag} / \mathrm{AgCl}$ reference electrode (RE) were applied directly to the membrane, 341 which acted as the working electrode (WE). Together with a platinum mesh counter electrode 342 (CE), the feed reservoir is essentially a standard three-terminal electrochemical cell. Inset is 343 an optical image of the membrane material. b, Steady-state diffusion curves obtained under 344 varied $\mathrm{V}_{\mathrm{g}}$ from $-0.5,-0.4$ to $-0.2 \mathrm{~V}$. c, Steady-state diffusion curves obtained under a 345 programmed $\mathrm{V}_{\mathrm{g}}$ sequence from $-0.2,-0.4,-0.5,0,0.2,0.4$ to $0.5 \mathrm{~V}$. The voltage changed 346 immediately after each potential step and was held for 90 minutes. The diffusion curves are

347 shown as the grey line, upon which linear regression was carried out for each $\mathrm{V}_{\mathrm{g}}$ and shown 348 as blue lines. Data were used after 70 minutes to allow diffusion to reach to the steady state.

349 d, Reversible modulation of ion diffusion with a $V_{g}$ whose values were varied between -0.5 $350 \mathrm{~V}$ and $0.2 \mathrm{~V}$ for multiple cycles. Inset (up) shows the membrane flux measured under 4 351 cycles of alternating $\mathrm{V}_{\mathrm{g}}$ over the course of 10 hours. Inset (down) is a zoom-in of the flux 352 transition as the $\mathrm{V}_{\mathrm{g}}$ changed from $-0.5 \mathrm{~V}$ to $0.2 \mathrm{~V}$ at the second cycle.

354 Fig. 2: Normalized membrane flux dependence on $V_{g}$ under various levels of 355 nanoconfinement and concentration gradient. Profiles of the modulated ion diffusion 
through charged layered graphene-based nanoporous membranes (with a $d$ of $2 \mathrm{~nm}$ ) measured

357 under different feed concentrations from a, $0.05 \mathrm{M}$ (black), b, $0.1 \mathrm{M}$ (black) to c, 0.5

$358 \mathrm{M}$ (black); and the profile dependence on nanoconfinement achieved via varying the

359 membrane interlayer spacing from $0.8 \mathrm{~nm}$ (yellow), $2.0 \mathrm{~nm}$ (black) to $5.4 \mathrm{~nm}$ (green). Ion

360 fluxes under various charging conditions were normalized to that obtained at zero volts vs.

$361 \mathrm{Ag} / \mathrm{AgCl}$ reference electrode. The electrolyte used was a $\mathrm{KCl}$ aqueous solution. The average

362 of three independent tests are plotted in the figures.

363

Fig. 3: Ion specific electrostatically modulated ion diffusion through layered graphene-

based nanoporous membranes $(d=2 \mathrm{~nm})$, a, Profiles of normalised membrane flux

dependence on $\mathrm{V}_{\mathrm{g}}$ measured in $\mathrm{KCl}, \mathrm{K}_{2} \mathrm{SO}_{4}, \mathrm{MgSO}_{4}$ and $\mathrm{MgCl}_{2}$ electrolytes and with a feed concentration of $50 \mathrm{mM}$. The average of three independent tests are plotted in the figures. $\mathbf{b}$,

Steady-state diffusion curves of sodium acetate (black), $\mathrm{KCl}$ (purple) and $\mathrm{NaCl}$ (blue) with a feed concentration of $0.5 \mathrm{M}$ under a $\mathrm{V}_{\mathrm{g}}$ sequence from -0.5 to $0.5 \mathrm{~V}$. The diffusion curves were shown as the grey dot lines, upon which linear regression was carried out for each $\mathrm{V}_{\mathrm{g}}$.

\section{Fig. 4: Role of ion-ion correlations in altering channel counter- and co-ion} concentrations, membrane potential and membrane flux against varied channel height.

a, the figure shows the normalized concentrations of both co- and counter-ion (inset) in an array of 2-nm graphene cascading nanoslits simulated with (solid) and without (open) ion-ion correlations contribution under various $\mathrm{V}_{\mathrm{g}}$. Feed concentrations were varied from $0.05 \mathrm{M}$ to 0.5 M. b, Membrane potential measured by experiment during steady-state, voltagemodulated diffusion against a varied $\mathrm{V}_{\mathrm{g}}$. The concentration gradient was created with a feed solution of $0.1 \mathrm{M}$, and a permeate solution of $1 \mathrm{mM} \mathrm{KCl}$. Inset shows the membrane potential obtained from continuum simulation based on PNP model (empty purple circles) and PNP/IC model (filled purple circles). c, the figure shows the corresponding $J-\mathrm{V}_{\mathrm{g}}$ relationships in 2$\mathrm{nm}$ graphene nanoslits. d, the figure shows the effect of channel size on flux enhancement simulated with $\mathrm{V}_{\mathrm{g}}=-0.3 \mathrm{~V}$ and a feed concentration of $0.05 \mathrm{M}$. The inset shows the 
384 cascading graphene nanoslits model geometry used for simulation. Reservoir length $\left(L_{\text {res }}\right)$, 385 nanoslit membrane length $\left(L_{\mathrm{mem}}\right)$, the lateral length of graphene sheet $\left(L_{\mathrm{G}}\right)$, sheet-to-sheet 386 aperture size $(\delta)$ and interlayer spacing $(d)$ were $100,80,55,2$ and $2 \mathrm{~nm}$ respectively. These 387 parameters were adopted from previous work in Ref. ${ }^{17}$. The lower range of the medium 388 dielectric constant $\left(\epsilon_{\mathrm{d}}\right)$ was set to be 10 inside the graphene nanochannels with a size of 2 $389 \mathrm{~nm}$, and was determined by a separate Molecular Dynamics (MD) simulation (Supplementary 390 Fig. 9). The correlation length $\left(l_{c}\right)$ in the PNP/IC model was set to be $0.66 \mathrm{~nm}$ which is the 391 size of hydrated $\mathrm{K}^{+}$and $\mathrm{Cl}^{-}$ions.

\section{Methods}

$394 \quad$ Fabrication of the layered graphene-based nonporous membranes

395 The layered graphene-based nanoporous membranes were prepared via direct-flow filtration 396 of chemically converted graphene colloids. Chemically converted graphene (CCG) 397 dispersions were synthesized following the method previously described in Ref. ${ }^{30}$. Briefly, 398 graphene oxide colloid $(0.5 \mathrm{mg} / \mathrm{ml}, 100 \mathrm{ml})$ prepared with the modified Hummers' method 399 was initially mixed with $0.2 \mathrm{ml}$ hydrazine ( $35 \mathrm{wt} \%$ in water) and $0.35 \mathrm{ml}$ ammonia (28 wt\% 400 in water) solution in a glass flask. After being vigorously shaken for a few minutes, the flask 401 was put into a water bath $\left(\sim 100^{\circ} \mathrm{C}\right)$ for 3 hours. A controlled amount of the as-obtained CCG 402 dispersion was vacuum filtrated through a mixed cellulose ester filter membrane $(0.05 \mu \mathrm{m}$ 403 pore size). The vacuum was disconnected immediately after all free CCG dispersion was 404 gone from the filtrate cake, at which a wet gel membrane remained. The gel membrane was 405 then carefully peeled from the filter, immediately transferred to a Petri dish and immersed in 406 deionized water overnight to further remove the remaining ammonia and unreacted 407 hydrazine. To ensure sufficient mechanical robustness and integrity of the freestanding 408 membranes, the CCG mass loading of all wet gel membrane samples was controlled to be 1 $409 \mathrm{mg} / \mathrm{cm}^{2}$ (A schematic of graphene membrane preparation was shown in Supplementary Fig. $4101)$.

411 Varying the interlayer spacing of the layered graphene-based nanoporous membranes

412 Capillary compression method was used, described in Ref. ${ }^{16}$, and shown in the 413 Supplementary Fig.1, to tune the average interlayer spacing of the nanoporous graphene 414 membranes. Briefly, the water inside the as-assembled gel membranes was first exchanged 
415 with a controlled ratio of volatile/non-volatile miscible solution (water/ sulphuric acid 416 solution in this case). The volatile liquid inside the gel membrane was then selectively 417 removed via vacuum evaporation. The removal of the volatile component of the miscible 418 solution exerted capillary compression between CCG layers, leading to a uniform shrinkage 419 of membrane thickness and thereby decreasing the average interlayer spacing in a collective 420 manner. As the non-volatile part of the miscible solution in the gel membrane remained, the 421 average interlayer spacing could be readily tuned by adjusting the ratio of volatile/non-

422 volatile solutions. Subsequently, the as-compressed gel membranes were washed thoroughly 423 with deionized water, exchanging the non-volatile liquid back to water prior to test. It is 424 worthwhile to point out that this exchange of non-volatile back to water step would cause a 425 slightly increased membrane thickness. The average interlayer spacing, $d$, was estimated 426 following the equation:

\section{$d=\frac{\text { Areal mass density of graphene } \times \text { thickness of the gel membranes }}{\text { Areal mass loading of the gel membranes }}$}

427 in which, the thickness of the gel membranes was measured after the final "exchange of 428 liquid" step (Supplementary Fig. 1).

429

Measurement of ion diffusion across charged nanoporous graphene membranes

431 As-prepared layered graphene-based nanoporous membranes were mounted between two 432 clamp holders (an image shown in the Supplementary Fig. 1), where one side of the 433 membrane was attached to a platinum ring, making the membrane the working electrode in a 434 three-terminal electrochemical cell. Two compartments, namely the feed and permeate, were 435 then connected to each side of the membrane holder, constituting a standard configuration for 436 measuring membrane permeability. The feed compartment was filled with $0.05,0.1$ and 0.5 $437 \mathrm{M}$ electrolyte solutions to be individually tested, while the permeate was filled equivalent 438 volume of deionized water with conductivity less than $4 \mu \mathrm{S} / \mathrm{cm}$. A constant potential within a 439 range from $-0.5 \mathrm{~V}$ to $0.5 \mathrm{~V}$ was generated using a BioLogic VMP-300 system while the ion 440 permeation through the membranes was constantly monitored by a conductivity meter. Both 441 counter (a platinum mesh) and $\mathrm{Ag} / \mathrm{AgCl}$ reference electrodes were placed at fixed positions in 442 the feed compartment, close to the membrane surface to minimize Ohmic loss. The 443 conductivity change in the permeate reservoir was plotted against elapsed time to give the 
444 diffusion curve. To ensure the concentration gradient between feed and permeate reservoirs 445 remained constant throughout the diffusion measurement, the conductivity variance of the 446 feed reservoir was monitored before and after the diffusion test. For the lowest feed 447 concentration of $0.05 \mathrm{M}$, the feed conductivity values before and after the diffusion at the 448 highest membrane potential of $-0.5 \mathrm{~V}$ were $6.31 \mu \mathrm{S} / \mathrm{cm}$ and $6.21 \mu \mathrm{S} / \mathrm{cm}$, respectively. The 449 decrease in feed solution concentration was less than $2 \%$. To further ensure that the 450 concentration difference between the feed and permeate reservoirs remained constant 451 throughout the diffusion test, no readings were made after the concentration in the permeate 452 reached $1.4 \times 10^{-4} \mathrm{M}(\sim 20 \mu \mathrm{S} / \mathrm{cm}$ at room temperature $)$. Solutions in both reservoirs were 453 constantly circulated, to avoid possible concentration gradient build-up at membrane 454 surfaces.

455 A variety of ions were tested to see if their fluxes through the layered graphene membrane 456 could also be modulated by the applied potential. In order to properly examine and compare 457 the effect of different ion pairs, our choices are mainly focused on simple alkali metal cations 458 including $\mathrm{Li}^{+}, \mathrm{Na}^{+}, \mathrm{K}^{+}$and $\mathrm{Cs}^{+}$then paired with $\mathrm{Cl}^{-}$and $\mathrm{SO}_{4}{ }^{2-}$, in particular for a known 459 strong ion-ion correlations of $\mathrm{SO}_{4}{ }^{2-}$ with cations ${ }^{27}$.

460

461 Because of the micro-corrugated molecular configuration of $\mathrm{CCG}^{31}$, it is difficult to gain 462 meaningful information on the average interlayer spacing of the membrane during diffusion 463 testing $^{16}$. We measured the membrane thickness variation in-situ as an indicator for any 464 structural deformation of the membrane during charging. Additionally, the layered 465 nanoporous graphene-based membrane can retain a stable structure under both negatively and 466 positively polarised as revealed by thousands of electrochemical cycling experiments ${ }^{16}$.

467

468 Mean-field theory modeling of ion diffusion through charged graphene nanochannels

469 To gain further insights into the experimentally observed voltage-modulated ion diffusion 470 phenomenon, we carried out simulations to research into the effect of the interplay among 471 various interactions in nanoconfined fluids. We used a modified Poisson-Nernst-Planck 472 model (described as PNP model in this work) to simulate ion diffusion through charged 473 graphene nanochannels, which took into account the ion steric effect in solutions with high 474 concentrations of $\sim 1 \mathrm{M}$ and under high potentials $\left(\phi \gg \frac{k_{B} T}{z e}\right)^{19,32}$. The solution for a binary 
475 symmetric electrolyte e.g. $\mathrm{KCl}$, whose cation and anion were of nearly the same size was 476 presented as follows,

$$
\begin{gathered}
\epsilon_{0} \epsilon \nabla^{2} \phi=-\rho=-\sum_{i=i}^{N} z_{i} e N_{A} n_{i} \\
\frac{\partial n_{i}}{\partial t}=D_{i} \nabla^{2} n_{i}+e z_{i} \mu_{i} n_{i} \nabla \phi+\frac{D_{i} N_{A} a^{3} n_{i} \nabla\left(n_{+}+n_{-}\right)}{1-N_{A} a^{3}\left(n_{+}+n_{-}\right)}
\end{gathered}
$$

477 where $a=0.66 \mathrm{~nm}$, the size of both cation and anion of $\mathrm{KCl}$ aqueous electrolyte; $\epsilon_{0}$ and $\epsilon$ 478 were the vacuum and relative permittivity of solution; $\phi$ was the electric potential 479 distribution; $\rho$ was the net charge density; $N_{A}$ was the Avogadro constant; $D_{i}, \mu_{i}, z_{i}, n_{i}$ were 480 diffusivity, electro-mobility, valence number and concentration distribution for species $i$, 481 respectively. The diffusivity and electro-mobility of $\mathrm{K}^{+}$and $\mathrm{Cl}^{-}$ions were considered the 482 same $^{14}$. The last term of Eq.3, also called the entropy term, was added as a correction for 483 finite ion size. This was to ensure that the maximum concentration of ions $\left(n_{+}+n_{-}\right)$in 484 reservoirs did not $n_{\max }=1 / N_{A} a^{324}$.

485 A 40-layer parallel-aligned graphene array shown in the Supplementary Fig. 5 (representing 486 the structure of the graphene membrane used in experiment) was sandwiched in between two 487 100-nm-in-length feed and permeate reservoirs. We adopted the optimized geometries of the 488 graphene array from our previous work in which the three critical parameters are the length 489 of graphene sheet $L_{\mathrm{G}}=55 \mathrm{~nm}$, channel height (interlayer spacing) $d=2 \mathrm{~nm}$ and in-plane 490 aperture size $\delta=2 \mathrm{~nm}^{14}$. Given the symmetry of the channel array structure, we built and 491 studied only half of the minimal unit cell. An electric potential difference of $\mathrm{V}_{\mathrm{g}}=0 \sim 0.3 \mathrm{~V}$ 492 between $\Gamma_{1}$ and $\Gamma_{G}$ was imposed to mimic the gate voltage $\left(\mathrm{V}_{\mathrm{g}}\right)$ applied in the experiment. 493 Impermeable boundary conditions were employed at both the entrance of $\Gamma_{1}$ and $\Gamma_{4}$ for ionic 494 concentration and electric field. Feed concentrations in the feed reservoir $(0.05,0.01$ to $0.5 \mathrm{M}$ 495 as used in experiments) were fixed at the entrance $\Gamma_{1}$, and the concentrations of cations (or 496 anions) at the permeate exit $\Gamma_{4}$ were set as $1 \times 10^{-5} \mathrm{M}$. To simulate the stationary state of 497 diffusion as reflected by a constant membrane permeability observed in experiment, and the 498 dilute solution in the permeate reservoir, the flux of both cations and anions at the exit $\Gamma_{4}$ 499 were set as equal. 
500 The effect of a possible decrease in dielectric constant arising from strong nanoconfinement ${ }^{33}$ 501 was investigated via varying the dielectric constant $\epsilon_{h}$ in $\Omega_{2}$ from 78.5 to 5 , while the 502 dielectric constant in $\Omega_{1}$ and $\Omega_{3}$ remained constant at 78.5. The dielectric constant of

503 graphene $\left(\epsilon_{G}=4\right)$ was the same as our previous work ${ }^{34}$. The average ionic fluxes at the exit 504 of permeate side were sampled and compared among those under varied potential conditions 505 and concentration differences. The results are summarized in the Supplementary Fig. 6. All 506 simulations were carried out with the COMSOL software package.

508 We further considered the effect of a possible increase in the intrinsic ion mobility when 509 subjected to severe nanoconfinement and with an externally applied electric field on 510 enhanced channel permeability across $\Omega_{2}{ }^{3}$. The results of a $V_{g}=-0.2 \mathrm{~V}$ were shown in the 511 Supplementary Fig. 7. It can be seen that the variation of ion mobility can result in a higher 512 ionic flux. In a permeate electrolyte concertation of $0.05 \mathrm{M}$, the overall flux under a $\mathrm{V}_{\mathrm{g}}=$ $513-0.2 \mathrm{~V}$ became higher than that under a $\mathrm{V}_{\mathrm{g}}=0 \mathrm{~V}$ when the ion diffusivity was to increase by 514 a factor of 10. Nevertheless, such a sharp increase in ion diffusivity required to compensate 515 the suppression from co-ion exclusion on flux was very unlikely according to existing 516 nanofluidics theories ${ }^{35}$. Furthermore, the present model also indicated that the magnitude of 517 the diffusion flux enhancement was favourable for high feed concentrations $(\Delta n=0.5 \mathrm{M})$, 518 opposite of our experimental observations.

519 MD simulation of the electrical double layers confined in a 2-nm graphene nanochannel

521 MD simulation was further carried out to study in more detail the EDLs structure confined in 522 graphene nanochannels in equilibrium. A "H" shaped graphene channel structure was 523 constructed (Supplementary Fig. 9), and periodic boundary condition was applied making 524 any $5.7 \mathrm{~nm}$-length reservoir connected to a $5 \mathrm{~nm}$-length graphene nanochannel or vice versa. 525 The LJ parameters of ions and graphene atoms was taken from literature and the TIP4P526 PPPM water model ${ }^{36}$ was employed. Columbic interactions were calculated with a particle527 particle particle-mesh (PPPM) solver. During all simulations, the temperature was held 528 constant via a Berendsen thermostat at $300 \mathrm{~K}$ via the LAMMPS MD code. 
530 Initially, $1 \mathrm{M} \mathrm{KCl}$ solution and pure water were filled respectively in reservoir and 531 nanochannel. The Constant Charge Methods (CPM) ${ }^{17}$ were employed to vary the charge 532 density status of graphene atoms (green) from $0 \sim-150 \mathrm{mC} / \mathrm{cm}^{2}$. Counter-ions were added 533 into the reservoir to retain electroneutrality. Within the first $0.8 \mathrm{~ns}$, a layer of ghost atoms (at 534 the gate of nanochannel) blocked the entrance of ions, while allowing the exchange of water 535 between reservoirs and the nanochannel. Once the equilibrium was reached, the ghost atoms 536 were removed, allowing ions to diffuse freely into the nanochannel. Simultaneously, the 537 decrease in ionic concentration in the reservoir was compensated by ion insertion. For every $53810 \mathrm{fs}$, the reservoir concentration was checked and toped back up to $1 \mathrm{M}$ by inserting ion 539 pairs at the reservoir centers. When the equilibrium ( $>20 \sim 30 \mathrm{~ns})$ was reached, the 540 concentration check and ion insertion were turned off. The system configuration at 541 equilibrium for the last $7 \sim 8$ ns was dumped for post-analysis.

543 The ion concentration in reservoirs at the last stage was measured to be $1.02 \sim 1.05 \mathrm{M}$. It 544 should be noted that the enormous computation expense associated with simulating the 545 electrostatically modulated ion diffusion, specifically in term of a low feed concentration (50 $546 \mathrm{mM})$ and in a relatively long nanochannel $(10 \sim 100 \mathrm{~nm})$, made it impractical to carry out 547 such dynamic ion diffusion study directly using MD.

549 MD simulation was also used to quantify the variation of dielectric constant when confined in 550 a nanochannel with size of $2 \mathrm{~nm}$. The electric potential away from the graphene wall surface 551 was obtained by the integral of 1D Gauss' law. The contributions from graphene plus ions, 552 and the water molecules were calculated separately following the definition in our previous 553 work $^{17}$. Supplementary Fig. 9b showed the potential profiles of $\phi_{\text {ion }}$ and $\phi_{\text {water }}$ off the 554 channel wall and that of their combination $\phi_{n e t}$. The average dielectric constant of water 555 molecules was derived from $\epsilon_{d}=\phi_{\text {ion }} / \phi_{\text {net }}$. The calculation of the medium dielectric 556 constant gave a value of 10 , consistent with previous theoretical calculations and experiment

557 observation $^{28}$. This value was used in numerical simulation cases (for both PNP and PNP/IC 558 models).

559

560 Effect of ion correlations on diffusion through charged graphene nanochannels 
561 It is well acknowledged that EDLs rendered an oscillating layer structure within a few 562 nanometers off the electrode/electrolyte interface. Such an oscillating structure could be 563 generally explained by the presence of short-range ion correlations that led to effects of 564 "overscreening" 24 and "charge inversion" 25,26 at a charged electrode/electrolyte interface.

565 Given such an overscreening can result in a more concentrated co-ion layer, the incorporation 566 of an ion-correlation effect was investigated, as was its impact on ion diffusion properties 567 through a charged graphene nanochannel.

568

569 The model used was one developed recently by Bazant and colleagues ${ }^{24}$, and named as the 570 Poisson-Nernst-Planck model with Ion Correlation (PNP/IC) that shows better descriptions 571 especially on the oscillating EDLs structure than the previous PNP model. The governing 572 equations are,

$$
\begin{aligned}
& \epsilon_{0} \epsilon \nabla^{2} \phi(\mathbf{r})-\epsilon_{0} \epsilon l_{c}^{2} \nabla^{4} \phi(\mathbf{r})=-\rho(\mathbf{r})=-\sum_{i=i}^{N} z_{i} e N_{A} n_{i} \\
& \frac{\partial n_{i}}{\partial t}=D_{i} \nabla^{2} n_{i}+e z_{i} \mu_{i} n_{i} \nabla \phi(\mathbf{r})+\frac{D_{i} N_{A} a^{3} n_{i} \nabla\left(n_{+}+n_{-}\right)}{1-N_{A} a^{3}\left(n_{+}+n_{-}\right)} \quad \text { Eq.5 }
\end{aligned}
$$

573 Compared with the previous modified PNP model, an extra forth order Laplace operator was 574 added for the correction for ion-ion correlation and $l_{c}$ for correlation length.

575

576 The channel geometry and configuration were the same as shown in the Supplementary Fig. 577 5. According to the MD simulation study, the dielectric constant in $\Omega_{2}$ was set at $\epsilon_{d}=10$, 578 and ion size and correlation length given as $a=l_{c}=0.66 \mathrm{~nm}$ for aqueous $\mathrm{KCl}$ solution were 579 employed. The boundary conditions in PNP/IC modeling were the same as that in the 580 previous PNP model, and the equal flux of cation and anion was maintained at the exit as a 581 result of diffusion potential. The numeric implementation of PNP/IC model in COMSOL was 582 followed by the suggestion by Liu, et. al. ${ }^{37}$ and Xie, et. al. ${ }^{38}$.

583

584 Such a different flux - potential relationship revealed with the PNP/IC model was attributed 585 to the increase of the co-ion (anion) concentration as a result of the strong ion-ion correlation. 586 The average concentration in $\Omega_{2}$ over different applied channel potential was plotted in Fig. 587 4. At a low voltage range of $0 \sim 0.1 \mathrm{~V}$, the exclusion of co-ions resulted in a decrease of the 
588 overall anion concentration, thus the ion diffusion flux. When the applied potential increased, 589 the cation-anion correlations dominated the first-layer counter-ion screening, resulting in an 590 increase of both counter- and co-ion concentrations, and thus an enhanced ionic flux. It was 591 also seen that lower feed reservoir concentrations corresponded to a more significant EDL 592 structure change, reflected by a larger degree of variations in both co- and counter-ion 593 concentrations for a given channel potential. In addition, the result of various channel 594 dielectric constant $\left(\epsilon_{d}\right)$ from 78.5 to 10 in the PNP/IC model were also plotted in the 595 Supplementary Fig. 10, to show the effect of an increasing nano-confinement. Similar to the 596 claim by Bazant et. al. ${ }^{24}$, with the increase of dielectric constant, the screening of solvent 597 precedes the correlations among the ions, and the PNP/IC model reduces to the general PNP 598 model with an negligible contribution from the ion-ion correlations term in Eq. 4. However 599 under severe nanoconfinement, the ion-ion correlations effect has a significant influence on 600 the distribution and dynamics of ions in the EDL.

601

602 Although the PNP/IC model appeared to successfully reproduce most of the experimental 603 observations, it could not be definitely concluded that the origin of the as-observed, 604 experimental permeability enhancement could be attributed to the anomalously strong ion-ion 605 correlations. Rather, the results presented here suggest a basic research framework upon 606 which future understanding can be built by studying the effect of ion-ion correlations on ion 607 dynamics, particularly in nanoconfined aqueous liquid systems.

608

\section{Data availability}

610 The data that support the plots within this paper and other findings of this study are available 611 from the corresponding author upon reasonable request.

612

\section{References for the Methods}

614 30. Li, D., Muller, M. B., Gilje, S., Kaner, R. B., Wallace, G. G. Processable aqueous dispersions of graphene nanosheets. Nat. Nanotechnol. 3, 101-105 (2008).

616 water and potential application for nanofiltration. Chem. Commun. 47, 5810-5812 (2011). 
621 32. Kornyshev, A. A. Double-layer in ionic liquids: paradigm change? J. Phys. Chem. B

33. Ghoufi, A., Szymczyk, A., Renou, R., Ding, M. Calculation of local dielectric

34. Daiguji, H., Yang, P., Majumdar, A. Ion transport in nanofluidic channels. Nano Lett. 4, 137-142 (2003).

35. Daiguji, H. Ion transport in nanofluidic channels. Chem. Soc. Rev. 39, 901-911 (2010).

36. Horn, H. W. et al. Development of an improved four-site water model for biomolecular simulations: TIP4P-Ew. J. Chem. Phys. 120, 9665-9678 (2004).

37. Liu, J. L. Numerical methods for the Poisson-Fermi equation in electrolytes. J. Comput. Phys. 247, 88-99 (2013).

38. Xie, D., Liu, J. L., Eisenberg, B. Nonlocal Poisson-Fermi model for ionic solvent. Phys. Rev. E 94, 012114 (2016). 


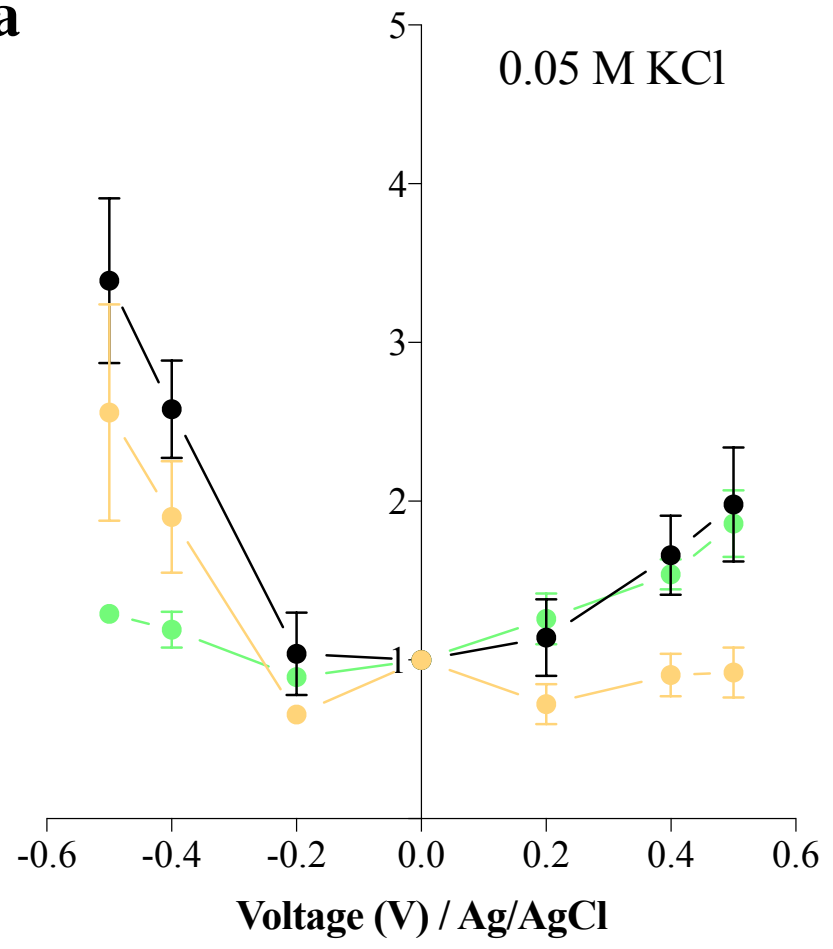

b

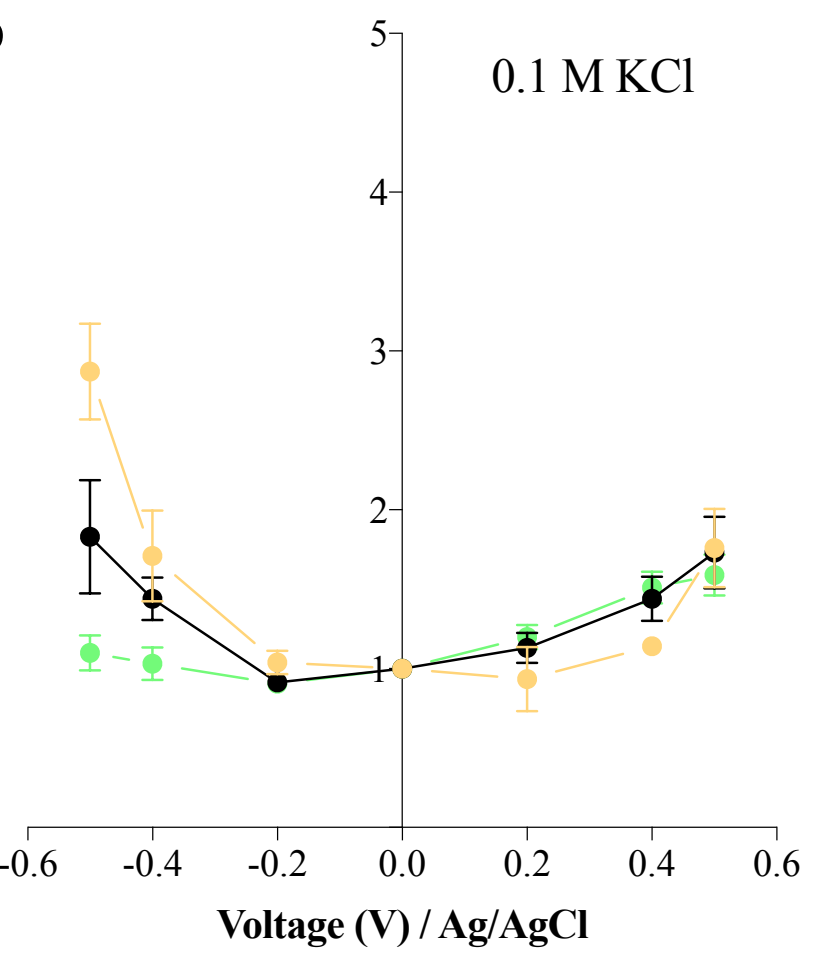

c

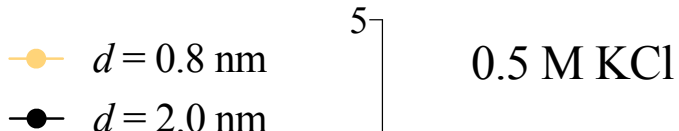

$\rightarrow-d=2.0 \mathrm{~nm}$ $d=5.4 \mathrm{~nm}$
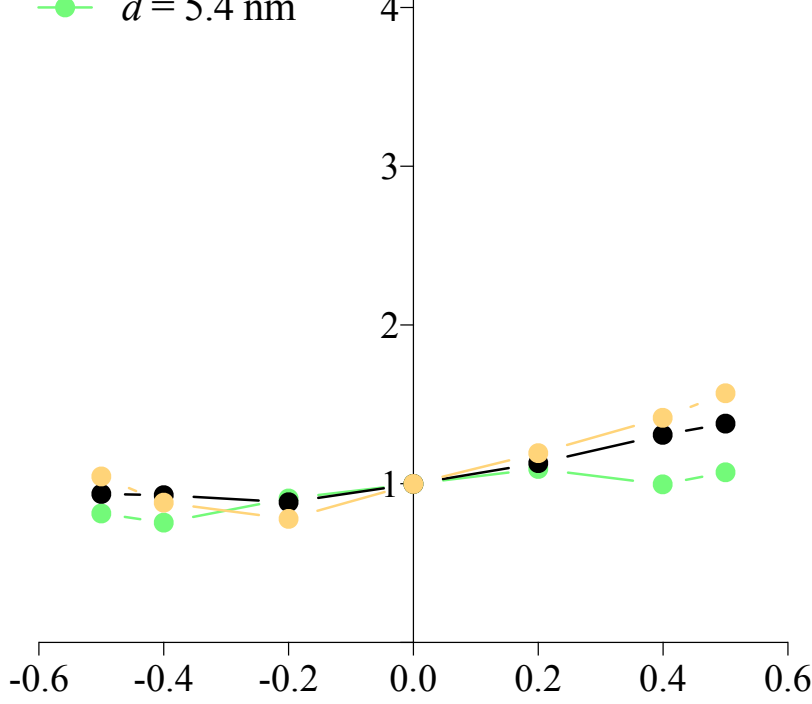

\section{Voltage (V) / Ag/AgCl}




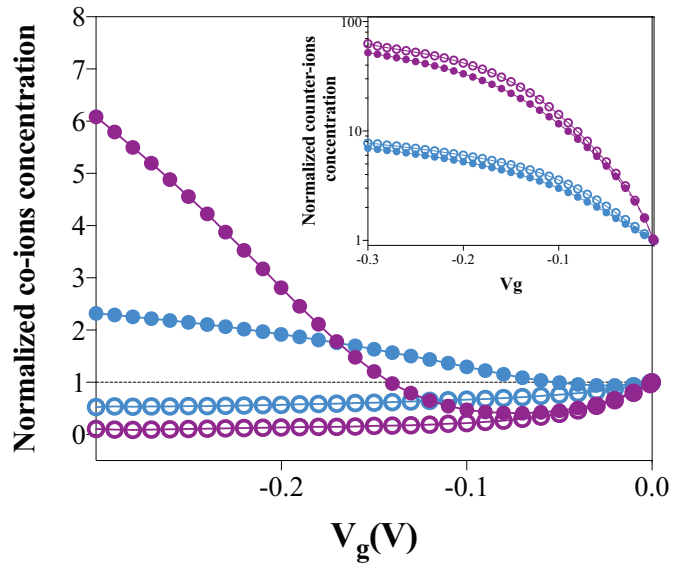

b

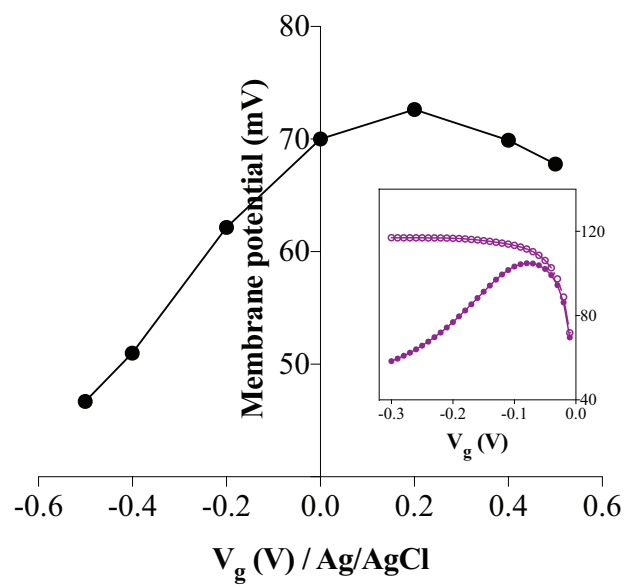

c

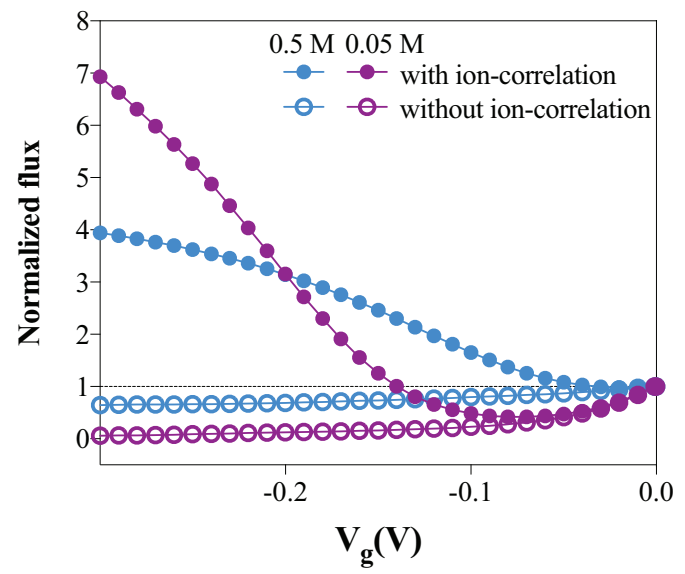

d

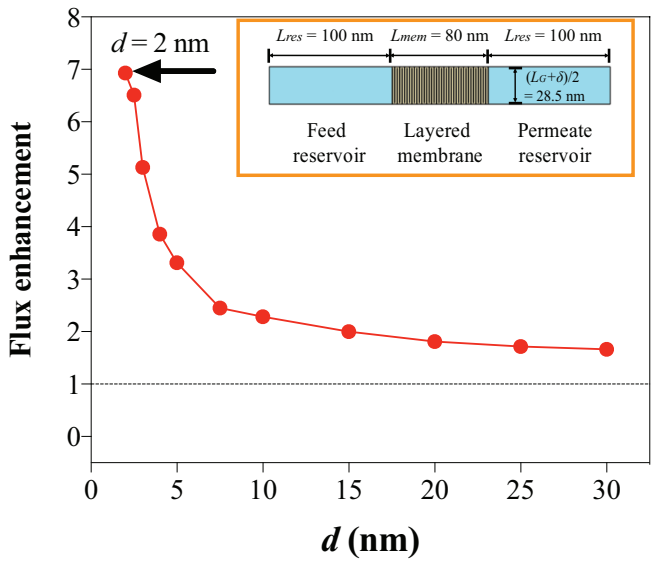

